\title{
Determining the composition of radio plasma via circular polarization: the prospects of the Cygnus A hot spots
}

\author{
Torsten A. Enßlin and Sebastian Hutschenreuter \\ Max Planck Institute for Astrophysics, Karl-Schwarzschildstr.1, 85741 Garching, Germany; \\ Ludwig-Maximilians-Universität München, Geschwister-Scholl-Platz 1, 80539 Munich, Germany \\ Gopal-Krishna \\ Aryabhatta Research Institute of Observational Sciences (ARIES), Manora Peak, Nainital - 263129, INDIA
}

\begin{abstract}
The composition of the relativistic plasma produced in active galactic nuclei and ejected via powerful jets into the interstellar/intergalactic medium is still a major unsettled issue. It might be a positron-electron plasma in case the plasma was created by pair production in the intense photon fields near accreting super-massive black holes. Alternatively, it might be an electron-proton plasma in case magnetic fields lift and accelerate the thermal gas of accretion discs into relativistic jets as the recent detection of $\gamma$-rays from blazars indicates. Despite various attempts to unambiguously establish the composition of the relativistic jets, this remains a major unknown. Here, we propose a way to settle the question via sensitive measurements of circular polarization (CP) in the radio emission of the hot spots of bright radio galaxies like Cygnus A. The $\mathrm{CP}$ of synchrotron emission is determined by the circular motions of the radiating relativistic leptons. In case of charge symmetric energy spectra of an electron-positron plasma, it should be exactly zero. In case of an electronproton plasma the electrons imprint their gyration onto the $\mathrm{CP}$ and we expect the hot spots of Cygnus A to exhibit a fractional CP at a level of $10^{-3}(\nu / \mathrm{GHz})^{-1 / 2}$, which is challenging to measure, but not completely unfeasible.
\end{abstract}

\section{INTRODUCTION}

\section{A. Circular polarization}

The radio synchrotron emission of relativistic plasma might exhibit a slight circular polarization (CP) due to the circular motions of the emitting leptonic particles, either electrons, or even electrons and positrons (see below). The radiation of relativistic electrons and positrons is beamed in the direction of their instantaneous motion and for this reason very little of the circular motion of an ensemble of relativistic leptons gets imprinted onto their radiation as circular polarization. Nevertheless, an asymmetry in the charge distribution of particles contributing to a given observational frequency should leave a weak CP signal. Such an asymmetry should exist if the radio plasma is mainly an electron-proton plasma, as one might expect in case the radio jets consist of material launched magnetically from accretion discs around super-massive black holes. On the other hand, no $\mathrm{CP}$ signal should arise in case radio plasma is composed of electrons and positrons created in similar amounts and with identical spectra by pair production events from hard photons in the vicinity of such black holes. Thereafter, acceleration and energy loss mechanism should not distinguish between electrons and positrons in case the radio plasma stays purely leptonic in its subsequent evolution. Then the dynamics of electrons and positrons is (mirror) symmetric and they should aquire similar energy spectra, which leads to cancellation of their individual $\mathrm{CP}$ contributions.

Therefore, the detection of CP intrinsically emitted from radio plasma would indicate that the charge symmetry of the synchrotron radiation arising from the plasma constituents is broken, and therefore either an electron proton plasma is dominant, or alternatively, the particle acceleration mechanism favours electrons or positrons for a yet unknown reason.
More interesting, therefore, would be a reliable observational upper bound on the CP flux from radio plasma, which is distinctly below the expected level for an electron-proton plasma. That would be a strong evidence against emission from an electron-proton plasma and therefore favor an electron-positron plasma.

Other sources of CP should be excluded before accepting the electron-proton plasma scenario via $\mathrm{CP}$ detection. The primary candidates for this are Faraday conversion and instrumental polarization.

Faraday conversion is the transformation of linearly polarized emission into CP by the different dispersion a linear polarized wave experiences depending on whether its polarization is parallel or perpendicular to the magnetic field. It is believed to be the main cause of the highly variable $\mathrm{CP}$ observed in compact objects [1 9] like Sagrittarius A*, GRS 1915, SS 433 [10 13. Faraday conversion can be identified via its characteristic dependence on wavelength to the third power, as long one does not probe the extreme regimes like in AGNs [14]. It operates most efficiently in the strong magnetic fields and dense plasma present near these objects. Thus, if we wish to minimise the contamination to $\mathrm{CP}$ on account of Faraday conversion, we should investigate regions with weaker magnetic field strengths and lower particle densities. As we still need a high brightness, the hot spots of radio galaxies seem to be ideal, and the brightest of those are the ones seen at the tips of the radio lobes of Cygnus A, which will therefore be the focus of the present theoretical study.

Instrumental polarization is a severe problem, given that the fractional CP is expected to be very small. Any uncorrected leakage of total intensity or linear polarization into CP channels could lead to a spurious detection. There are two possibilities to place limits on the level of instrumental polarization. The first is that the observation of many sources taken together 
should not show any preference for any one sign of the $\mathrm{CP}$, as this is determined by whether the magnetic field in the emitting region is pointing towards us or away from us and therefore should happen with equal frequency. Furthermore, the temporal evolution of magnetic field structures in hot spots of radio galaxies should be well beyond human timescales, so that a given hot spot should always exhibit the same CP. Thus, observations with different instruments having different systematics can allow us to gain confidence about the genuineness of an inferred CP signal and its sign. Secondly, in case Faraday rotation information for the hot spot plasma exists, the expected CP sign in case of an electron proton plasma will be known in advance. CP should exhibit a rotational sense that is opposite to that of the direction of the Faraday rotation [15]. An agreement between the expected and observed CP signs would provide confidence in the genuineness of the (intrinsic) CP signal, whereas a disagreement would be indicative of uncorrected instrumental polarization. Note, however, that an observed Faraday rotation most likely traces magnetic fields surrounding the hot spot. If the Faraday rotation is indeed intrinsic, then a charge asymmetry in at least the thermal plasma there is confirmed, since such an asymmetry is a necessary condition of the Faraday effect.

\section{B. Previous studies}

Previous proposals to settle the question of the composition of radio plasma were usually based on energetic arguments. More importantly, from an observational standpoint, the composition of the relativistic nonthermal plasma radiating in the jets and lobes of radio galaxies continues to be a long-standing issue (e.g., 16 19]). This question is crucial also because the acceleration and deceleration of the jet near a supermassive black hole depends on the composition of its plasma (e.g., 20 22]). Over the years, a number of authors have argued in favour of the dominance of relativistic electron-positron pair-plasma in the jets ejected by the central engines of radio galaxies, both from observational and theoretical perspectives (e.g., 16, 23 25]). More recent arguments for a significant, if not dominant, presence of relativistic pair plasma in the radio lobes include those put forward by Kawakatu et al. [26] and Potter [27] who also provide a summary of the observational and theoretical work done on this topic.

According to a currently popular scenario, the generation of relativistic pair-plasma begins with photonphoton pair production in the spark gap of the magnetosphere surrounding a supermassive black-hole $([20,28])$ and the process continues along the length of the jet, e.g. via dissipation of magnetic energy by reconnection (see, e.g., [27]). Although, at large distances from the black-hole, some hadronic plasma may gradually get entrained in the jet flow, this process is unlikely to be efficient at least in Fanaroff-Riley type II (FR II) radio galaxies, since their jets remain effectively shielded by the cocoon of relativistic lobe plasma [27, 29, 30]. For FR I radio galaxies, the need for non-radiating pressure support of the radio plasma hints at an efficient entrainment of protons into the jet [31].

A hadronic jet model was proposed by Mannheim and collaborators [32 35] to explain the TeV gammaray emission of blazars. The same emission can, however, also be explained in terms of leptonic models, which invoke synchrotron self-Compton (SSC) emission to produce $\mathrm{TeV}$ photons.

In the case of FR II radio sources, hadronic jets have been considered to explain their X-ray emission in terms of proton synchrotron radiation, rather than as the inverse-Compton boosted cosmic-microwave background photons [e.g., 36 40]. The needed high energy protons could even be injected into the jet via turbulent acceleration in the shear layer of the jet [4]. Alternatively, the x-ray jets could be synchrotron radiation produced by the secondary electrons arising from photo-hadronic interactions like the Bethe-Heitler process or photopion production [see 39, 42, 43. In all such scenarios of hadronic jets, one would expect neutrino emission from charged pion or neutron decay resulting from interactions between high energy protons and photons [44]. The recent observation of a high energetic neutrino from the Blazar TXS $0506+056$, is best explained by a hadronic emission process 45]. A similar signal has not yet been observed from FR II radio galaxies, which may constrain the hadronic jet model [46].

Lepto-hadronic model for high-energy emission from FR I radio galaxies are consistent with their observed high energy radiation [47].

Over the years, significant observational evidence has in fact emerged in support of a dominant leptonic relativistic plasma component in the extended radio lobes of FR II sources. The evidence has come mainly from balancing the observationally estimated pressure of the external X-ray emitting thermal plasma and the internal lobe pressure due to the nonthermal plasma estimated by modeling of the combined synchrotron/inverse-Compton spectrum of the lobe, as constrained by measurements at radio and $\mathrm{X}$-ray energies, respectively (e.g., [48 51]). This concurs with the conclusion reached indpendently for the lobes of several FR II sources, including Cygnus A, based on modeling of the observed shapes of the radio lobes (see [26]). The X-ray emission from hotspots of powerful FR II sources is often consistently explained in terms of the SSC mechanism, when the magnetic field is close to the equipartition value and under the assumption of an energetically significant, if not a dominant, pair-plasma (vis a vis the electron-proton plasma) [52, 53], which is in accord with the findings for powerful radio lobes of FRII sources (see above). Specifically, for the well studied case of Cygnus A, the analysis of the X-ray emission from its hotspots has lent strong support to such an interpretation [52, 54]. Clearly, pair-plasma dominated powerful hotspots would be entirely in tune with the dominance of pair-plasma inferred for FR II lobes (see above), since the latter are fed by the hotspots. Nonetheless, given the various uncertainties involved, such as 
spatial inhomogeneities in the radiating plasma and beaming of the radiation, independent observational constraints on the presence of an energetic proton population in the hotspots would be very desirable. A population of energetic protons in hot spots could be the origin of the ultra-high energetic cosmic rays observed, as the hot spots of FR II radio galaxies are potential acceleration sites (see e.g. [55] and reference therein). They would also be in line with the recent detection of neutrinos from the Blazar TXS 0506+056 [45].

There is a long history of circular polarization measurements aiming to determine the relativistic plasma composition near the jet's base itself. More recently, VLBI imaging of both circular and linear polarization have been carried out for a few blazars on sub-parsec scale, sometimes at multiple radio frequencies [56, 57. For the blazar 3C 279, Homan et al. [56] have reported a particularly detailed observational study which also includes simulations of their sensitive VLBI results in Stokes I, linear polarization, and circular polarization at 6 frequencies in the range 8 to $25 \mathrm{GHz}$. The significant detection of CP is thus interpreted by them primarily in terms of Faraday conversion of the linear polarization within the nuclear jet. Their main conclusion is that the jet is kinetically dominated by electron-proton plasma, though a significant presence of pair-plasma may still contribute to the radiation. Clearly, even this detailed analysis is vulnerable to uncertainties in the jet's physical parameters, like bulk speed and the minimum Lorenz factor of the relativistic plasma $\left(\gamma_{\min }\right)$. Interestingly, these uncertainties are largely obviated in the case of Cygnus A hotspots since not only is their motion non-relativistic (like all hotspots on kiloparsec-scale) but estimates of $\gamma_{\text {min }} \sim 600$ are also available, based on recent spectral turnover measurements made with the LOFAR telescope [58].

The energy of the leptons that are visible within the observationally accessible radio wavebands is usually not sufficient to explain the rough pressure balance between radio lobes and their surrounding thermal plasma. Additional relativistic protons could fill in the deficit. However, a large population of leptons with lower than observable energies could as well bridge the gap, as would deviations from the usually invoked equipartition assumption between particles and magnetic fields. For these reasons, a more direct determination of the composition of radio plasma via $\mathrm{CP}$ measurements as proposed here would be very important.

\section{ESTIMATION}

In the following we estimate the expected fractional $\mathrm{CP}$ emission arising from the hotspots of Cygnus A, in the case of a pure relativistic electron-proton plasma. A volume element of a hotspot along a line of sight (LOS) may harbor $n_{\mathrm{e}}$ relativistic electrons with a power law spectrum

$$
\frac{d n(\gamma)}{d \gamma}=\frac{n_{\mathrm{e}} \gamma^{-p}}{\gamma_{\min }^{1-p}-\gamma_{\max }^{1-p}}
$$

between $\gamma_{\min }$ and $\gamma_{\max }$ and with energy spectral index $p$. Their synchrotron emissivity is

$$
j=\left(\begin{array}{c}
j_{I} \\
j_{Q} \\
j_{U} \\
j_{V}
\end{array}\right)=\underbrace{j_{0} n_{\mathrm{e}} B_{\perp}^{\frac{p+1}{2}} \nu^{\frac{1-p}{2}}}_{j_{I}}\left(\begin{array}{c}
1 \\
q \\
u \\
v
\end{array}\right),
$$

where

$$
\begin{aligned}
j_{0} & =\frac{\mathrm{e}^{2}}{c}\left(\frac{\mathrm{e}}{2 \pi m_{\mathrm{e}} c}\right)^{\frac{p+1}{2}} \frac{3^{\frac{p}{2}}(p-1) \Gamma\left(\frac{3 p-1}{12}\right) \Gamma\left(\frac{3 p+19}{12}\right)}{2(p+1)\left(\gamma_{\min }^{1-p}-\gamma_{\max }^{1-p}\right)} \\
q & =-\frac{p+1}{p+7 / 3} \cos (2 \varphi) \\
u & =+\frac{p+1}{p+7 / 3} \sin (2 \varphi) \\
v & =-\frac{j_{1} B_{\|}}{\left(\nu B_{\perp}\right)^{1 / 2}} \\
j_{1} & =\frac{171}{250}\left(\frac{3 \mathrm{e} p}{2 \pi m_{\mathrm{e}} c}\right)^{1 / 2}=0.06268\left(\frac{p \mathrm{GHz}}{\mathrm{Gauss}}\right)^{1 / 2}
\end{aligned}
$$

and the magnetic field is

$$
\mathbf{B}=\left(\begin{array}{c}
B_{\perp} \cos (\varphi) \\
B_{\perp} \sin (\varphi) \\
B_{\|}
\end{array}\right)=\left(\begin{array}{c}
\sin (\theta) \cos (\varphi) \\
\sin (\theta) \sin (\varphi) \\
\cos (\theta)
\end{array}\right) B
$$

with the $z$-axis being parallel to the $\operatorname{LOS}$ and $\varphi$ the angle between the $x$-axis and the field component in the plane of the sky, $\mathbf{B}_{\perp}[59]$. The observational frequency is denoted by $\nu$. The symbols e, $m_{\mathrm{e}}$ and $c$ denote the elementary charge, the electron mass, and the speed of light, respectively. Relativistic positrons have exactly the same emissivities, with the only difference of an opposite Stokes $V$ sign. For this reason, there is no CP synchrotron emission in case the electron and positron spectra are identical in slope and normalization.

The total polarized emission of our electron-proton plasma is the volume integrated emissivity,

$$
J=\int_{\mathcal{V}} d x j(x) \approx \sum_{i=1}^{N_{\text {cell }}} \mathcal{V}_{i} j_{i}
$$

which we assume to be made of $N_{\text {cell }}$ similar sized cells with spatially constant emissivity $j_{i}$ within the cell volume $\mathcal{V}_{i} \approx \mathcal{V}_{\text {cell }}$.

For simplicity, we assume only the magnetic field orientation to vary from cell to cell, whereas the field strength $B$ and the electron spectrum $\frac{d n(\gamma)}{d \gamma}$ are taken to be approximately the same within the entire emitting volume $\mathcal{V}=N_{\text {cell }} \mathcal{V}_{\text {cell }}$. As no magnetic field direction is a priori preferred, we assume a random distribution of field orientations from cell to cell and therefore get for the average, dispersion, and root mean 
square (rms) of a quantity $X$

$$
\begin{aligned}
\bar{X} & =\frac{1}{4 \pi} \int_{0}^{2 \pi} d \varphi \int_{0}^{\pi} d \theta \sin \theta X(\theta, \varphi), \\
\sigma_{X}^{2} & =\frac{(X-\bar{X})^{2}}{N_{\text {cell }}}, \text { and } \\
X_{\mathrm{rms}} & =\sqrt{\overline{X^{2}}}=\sqrt{\bar{X}^{2}+\sigma_{X}^{2}} .
\end{aligned}
$$

The expected mean of the emission is $\bar{J}=$ $(\bar{I}, 0,0,0)^{\mathrm{t}}$, with $\bar{I}=\overline{j_{I}} \mathcal{V}$ and $\overline{j_{I}} \approx 0.719 j_{0} n_{\mathrm{e}} B^{\frac{3}{2}} \nu^{\frac{-1}{2}}$ for $p=2$. For an individual emission region like a hot spot, the different Stokes parameters show nonzero variance:

$$
\left(\begin{array}{c}
\sigma_{I} \\
\sigma_{Q} \\
\sigma_{U} \\
\sigma_{V}
\end{array}\right)=\left(\begin{array}{c}
0.190 \\
0.106 \\
0.106 \\
0.0229 B_{\mathrm{Gauss}}^{1 / 2} \nu_{\mathrm{GHz}}^{-1 / 2}
\end{array}\right) \frac{j_{0} n_{\mathrm{e}} B^{\frac{3}{2}} \nu^{\frac{-1}{2}} \mathcal{V}}{\sqrt{N_{\text {cell }}}}
$$

One should note that the assumption of randomness in the directional distribution is related to the cell sizes and the typical coherence length of the magnetic field, which may be rather large in Cygnus A due to the presence of shocks. In the most extreme limit of a completely coherent magnetic field one may set $N_{\text {cell }}=1$, in order to get a intuition for this effect.

In any case, the cell model is just a simplification, which may be dropped for a more elaborate calculation based on a physical model. It should, however, provide the right order of magnitude of the expected effect in a statistical sense.

If we investigate the vector $J^{\prime}=(I, P, V)^{\mathrm{t}}$ with $P=\sqrt{Q^{2}+U^{2}}$ being the total linear polarization, we find $\overline{J^{\prime}}=\left(1, \frac{p+1}{p+7 / 3} \frac{1}{N_{\text {cell }}}, 0\right)^{\mathrm{t}} \bar{I}$, since each cell has a fractional polarization of $\frac{p+1}{p+7 / 3} \approx 0.69$. Thus, we expect

$$
\frac{\sigma_{V}}{\sigma_{P}}=\frac{\sigma_{V}}{\sqrt{\sigma_{Q}^{2}+\sigma_{U}^{2}}}=0.153 B_{\mathrm{Gauss}}^{1 / 2} \nu_{\mathrm{GHz}}^{-1 / 2}
$$

Given that the fractional linear polarization of the Cygnus $\mathrm{A}$ hot spots is about $f_{P}=\sigma_{P} / \bar{I} \approx 0.7$ [60] and and the field strength is $B \approx 1.510^{-4}$ Gauss [61], we expect a fractional circular polarization of about $f_{V}=\sigma_{V} / \bar{I} \approx 1.31 \cdot 10^{-3} \nu_{\mathrm{GHz}}^{-1 / 2}$.

In order to be able to correctly assess the expeced $\mathrm{CP}$ emission, we must also consider the Faraday conversion effect of the foreground, which translates linear into circular polarized emission.

From the generic radiative transfer equations we know that

$$
j_{V, \mathrm{conv}}=\phi_{\mathrm{c}} \lambda^{3} j_{Q}
$$

where $\phi_{\mathrm{c}}$ is called the conversion measure in analogy to the Faraday rotation measure $\phi_{\mathrm{r}}$. In the thermal regime it can be sufficiently fitted via 62.

$$
\phi_{\mathrm{c}} \approx \frac{\mathrm{e}^{4}}{4 \pi^{2} m_{\mathrm{e}}^{3} c^{6}} \int_{\mathrm{LOS}} d r n_{\mathrm{th}} B_{\perp}^{2}
$$

under the assumption that the $j_{Q}$ emission takes place only in the hotspots. The thermal electron density $n_{\text {th }}$ poses a problem, as we do not have precise information on the electron column density of the Cygnus cluster in the vicinity of the hotspots, where most of the conversion is likely to take place. We do, however, have precise measurements on the Faraday rotation $\phi_{\mathrm{r}}$, which contains the same $n_{\mathrm{th}}$-dependence. We can write

$$
\phi_{\mathrm{c}} \approx \frac{\mathrm{e} B_{\mathrm{ICM}}}{2 \pi m_{\mathrm{e}} c^{2}} \int_{\mathrm{LOS}} d r \frac{\mathrm{e}^{3}}{2 \pi m_{\mathrm{e}}^{2} c^{4}} n_{\mathrm{th}} B_{\mathrm{ICM}} \approx \frac{\mathrm{e} c B_{\mathrm{ICM}}}{2 \pi m_{\mathrm{e}} c^{2}} \phi_{\mathrm{r}}
$$

under the assumption of a similar strength and correlation structure of the intracluster magnetic field in both the parallel and perpendicular component of the line of sight. We choose $B_{\mathrm{ICM}}=8 \cdot 10^{-6} \mathrm{G}$ as a typical value of the intracluster magnetic field [63]. Cygnus A has very high values of rotation measures with around $\left|\phi_{\mathrm{r}}\right| \approx \frac{\rho_{\phi}}{\lambda^{2}} \approx 1000 \frac{\mathrm{rad}}{\mathrm{m}^{2}}$ [64], which we will choose for our estimate. Therefore we arrive at $\phi_{c} \lambda^{3} \approx 2.013 \cdot 10^{-6} \nu_{\mathrm{GHz}}^{-3}$.

The mean $\overline{V_{\mathrm{c}}}$ is again zero. For the variance we find

$$
\sigma_{V_{\mathrm{c}}}=\phi_{\mathrm{c}} \lambda^{3} \sigma_{Q} \approx 2.133 \cdot 10^{-7} \frac{\bar{I}}{\nu_{\mathrm{GHz}}^{3} \sqrt{N_{\text {cell }}}}
$$

and for the ratio

$$
\frac{\sigma_{V_{\mathrm{c}}}}{\sigma_{V}} \approx 9.32 \cdot 10^{-6} \frac{1}{B_{\mathrm{Gauss}}^{1 / 2} \nu_{\mathrm{GHz}}^{2.5}} .
$$

Although small in the $\mathrm{GHz}$ regime, the above ratio reaches unity rather fast for smaller frequencies. We expect $\sigma_{V_{\mathrm{c}}} / \sigma_{V} \approx 1$ for $\nu \approx 57 \mathrm{MHz}$ and the aforementioned hotspot magnetic field strength of $B \approx$ $1.510^{-4}$ Gauss. Therefore, observations well above 56 $\mathrm{MHz}$ should not be significantly affected by Faraday conversion.

\section{CONCLUSIONS}

An electron-proton jet is expected to give rise to a fractional $\mathrm{CP}$ at a level of $10^{-3}(\nu / \mathrm{GHz})^{-1 / 2}$. Clearly, this is very challenging to measure, given the faintness of the signal and the systematic cross talk of polarized radio receivers. Nevertheless, systematic effects change from receiver to receiver, and even as a function of time as the relative orientation of sky and the telescope changes with the Earth's rotation. This engenders some hope that genuine $\mathrm{CP}$ detection might become technically feasible.

Due to the effect of Faraday conversion of LP into $\mathrm{CP}$, we expect the optimal frequency window for the detection of $\mathrm{CP}$ to be around $70 \mathrm{MHz}$. This renders low frequency radio telescopes like LOFAR most suitable. However, an excellent polarimetry is important as well, so that the the JVLA and in future the Square Kilometer Array as well as its precursors, the MeerKAT and ASKAP telescopes, are promising. The necessary polarimetric accuracies have already been achieved in observations [65]. 
A detection of the CP signal and thereby a confirmation of the electron-proton jet scenario might therefore be in reach soon. This would be very exciting, as it would contradict the prevailing majoritarian view in the radio galaxy community on jet composition in FR II radio galaxies. At the same time, it would make it more plausible that such galaxies are the acceleration sites of ultra high energy cosmic rays and it would also be in line with the recent detection of neutrinos from the Blazar TXS 0506+056 45]. The exclusion of electron-positron jets would, however, not be absolute. A possible scenario in which such jets can produce CP is the case of differing electron and positron energy spectra. These could be caused by their acceleration in a charge asymmetric environment, for example due to the entrainment of thermal electronproton plasma into the particle acceleration sites, so that plasma physical effects might engender different acceleration efficiencies of electrons and positrons.

A non-detection with a sensitivity well below the CP flux level estimated here for the hotspots of Cygnus A would also be very interesting, as it would argue strongly in favour of an electron-positron pair plasma, where both electrons and positrons have similar energy spectra, indicating a charge symmetric genesis and acceleration history for both species. The exclusion of the electron-proton jet scenario would be relatively firm in this case, as the only remaining way to avoid predicting detectable CP emission would be that the magnetic field in the hotspots points in a highly matched fashion towards and away from the observer, such that the CP emission of those volumes cancel. Given the high linear polarisation of the Cygnus A hotspots, and therefore an imbalance of the field orientations projected on the plane of the sky, a symmetry along the line of sights seems unlikely. Spatially resolved CP observations, or the study of a larger sample of hotspots of FR II galaxies could negate such an explanation.

To conclude, we have shown that sensitive CP observations of radio galaxy hotspots are a promising way to determine the composition of synchrotron plasma in radio galaxies in a way which is independent of many of the assumptions made in other lines of investigation probing this question.

\section{ACKNOWLEDGMENTS}

We would like to thank the organizers of the workshop Plasma universe and its structure formation at the Inter-University Centre for Astronomy and Astrophysics, Pune, India in 2017 for the stimulating atmosphere that led to this research. Furthermore, we thank an anonymous referee for constructive comments and Richard Anantua and Razieh Emami for pointing out an error in the original manuscript that is corrected here.
[1] T. W. Jones and S. L. Odell, ApJ 214, 522 (1977).

[2] T. W. Jones and S. L. Odell, ApJ 215, 236 (1977).

[3] P. E. Hodge, ApJ 263, 595 (1982).

[4] E. Valtaoja, Ap\&SS 100, 227 (1984).

[5] M. M. Komesaroff, J. A. Roberts, D. K. Milne, P. T. Rayner, and D. J. Cooke, MNRAS 208, 409 (1984).

[6] C.-I. Bjornsson, MNRAS 242, 158 (1990).

[7] T. Beckert and H. Falcke, A\&A 388, 1106 (2002), astro-ph/0112398.

[8] M. Ruszkowski and M. C. Begelman, ApJ 573, 485 (2002), astro-ph/0112090.

[9] T. A. Enßlin, A\&A 401, 499 (2003), astroph/0212387.

[10] G. C. Bower, H. Falcke, and D. C. Backer, ApJ 523, L29 (1999), astro-ph/9907215.

[11] R. J. Sault and J.-P. Macquart, ApJ 526, L85 (1999), astro-ph/9910052.

[12] R. Fender, D. Rayner, R. Norris, R. J. Sault, and G. Pooley, ApJ 530, L29 (2000), astro-ph/9911425.

[13] R. P. Fender, D. Rayner, D. G. McCormick, T. W. B. Muxlow, G. G. Pooley, R. J. Sault, and R. E. Spencer, MNRAS 336, 39 (2002), astro-ph/0204442.

[14] V. M. Vitrishchak and D. C. Gabuzda, International Journal of Modern Physics D 17, 1531 (2008).

[15] T. A. Enßlin, S. Hutschenreuter, V. Vacca, and N. Oppermann, Phys. Rev. D 96, 043021 (2017), 1706.08539.

[16] J. F. C. Wardle, D. C. Homan, R. Ojha, and D. H. Roberts, Nature 395, 457 (1998).

[17] M. Sikora and G. Madejski, ApJ 534, 109 (2000), astro-ph/9912335.

[18] P. D. Noerdlinger, Physical Review Letters 41, 135 (1978).
[19] D. S. De Young, ApJ 648, 200 (2006), astro$\mathrm{ph} / 0605734$.

[20] R. D. Blandford and R. L. Znajek, MNRAS 179, 433 (1977).

[21] R. D. Blandford and D. G. Payne, MNRAS 199, 883 (1982).

[22] E. S. Phinney, MNRAS 198, 1109 (1982).

[23] W. Kundt and Gopal-Krishna, Nature 288, 149 (1980).

[24] C. S. Reynolds, A. C. Fabian, A. Celotti, and M. J. Rees, MNRAS 283, 873 (1996), astro-ph/9603140.

[25] K. Hirotani, S. Iguchi, M. Kimura, and K. Wajima, ApJ 545, 100 (2000), astro-ph/0005394.

[26] N. Kawakatu, M. Kino, and F. Takahara, MNRAS 457, 1124 (2016), 1601.00771.

[27] W. J. Potter, MNRAS 473, 4107 (2018), 1709.09188.

[28] P. Goldreich and W. H. Julian, ApJ 157, 869 (1969).

[29] A. Mizuta, S. Yamada, and H. Takabe, ApJ 606, 804 (2004), astro-ph/0402355.

[30] P. A. G. Scheuer, MNRAS 166, 513 (1974).

[31] J. H. Croston, J. Ineson, and M. J. Hardcastle, MNRAS 476, 1614 (2018), 1801.10172.

[32] K. Mannheim, Space Sci. Rev. 75, 331 (1996), astro$\mathrm{ph} / 9502085$.

[33] K. Mannheim, Science 279, 684 (1998), astro$\mathrm{ph} / 9803241$.

[34] K. Mannheim, P. L. Biermann, and W. M. Kruells, A\&A 251, 723 (1991).

[35] K. Mannheim and P. L. Biermann, A\&A 253, L21 (1992).

[36] F. A. Aharonian, MNRAS 332, 215 (2002), astroph/0106037.

[37] Y. S. Honda and M. Honda, ApJ 613, L25 (2004), 
astro-ph/0408104.

[38] Y. Uchiyama, C. M. Urry, C. C. Cheung, S. Jester, J. Van Duyne, P. Coppi, R. M. Sambruna, T. Takahashi, F. Tavecchio, and L. Maraschi, ApJ 648, 910 (2006), astro-ph/0605530.

[39] W. Bhattacharyya and N. Gupta, ApJ 817, 121 (2016), 1511.00258.

[40] P. Breiding, E. T. Meyer, M. Georganopoulos, M. E. Keenan, N. S. DeNigris, and J. Hewitt, ApJ 849, 95 (2017), 1710.04250.

[41] M. Ostrowski, MNRAS 312, 579 (2000), astroph/9910491.

[42] M. Petropoulou, G. Vasilopoulos, and D. Giannios, MNRAS 464, 2213 (2017), 1608.07300.

[43] M. Kusunose and F. Takahara, ApJ 835, 20 (2017), 1611.08046.

[44] K. Mannheim and P. L. Biermann, A\&A 221, 211 (1989).

[45] I. Collaboration, ArXiv e-prints (2018), 1807.04300.

[46] A. Neronov, D. V. Semikoz, and K. Ptitsyna, A\&A 603, A135 (2017), 1611.06338.

[47] M. Reynoso, C. Medina, and G. Romero, 531, A30 (2011).

[48] J. Ineson, J. H. Croston, M. J. Hardcastle, and B. Mingo, MNRAS 467, 1586 (2017), 1701.05612.

[49] M. J. Hardcastle, M. Birkinshaw, R. A. Cameron, D. E. Harris, L. W. Looney, and D. M. Worrall, ApJ 581, 948 (2002), astro-ph/0208204.

[50] J. H. Croston, M. Birkinshaw, M. J. Hardcastle, and D. M. Worrall, MNRAS 353, 879 (2004), astro$\mathrm{ph} / 0406347$.

[51] C. Konar and M. J. Hardcastle, MNRAS 436, 1595 (2013), 1309.1401.

[52] M. J. Hardcastle, D. E. Harris, D. M. Worrall, and M. Birkinshaw, ApJ 612, 729 (2004), astro$\mathrm{ph} / 0405516$.

[53] J. Kataoka and E. Stawarz, ApJ 622, 797 (2005), astro-ph/0411042.

[54] A. S. Wilson, A. J. Young, and P. L. Shopbell, ApJ 544, L27 (2000), astro-ph/0009308.

[55] B. Eichmann, J. P. Rachen, L. Merten, A. van Vliet, and J. Becker Tjus, JCAP 2, 036 (2018), 1701.06792.

[56] D. C. Homan, M. L. Lister, H. D. Aller, M. F. Aller, and J. F. C. Wardle, ApJ 696, 328 (2009), 0902.0810.

[57] D. C. Gabuzda, V. M. Vitrishchak, M. Mahmud, and S. P. O'Sullivan, MNRAS 384, 1003 (2008), 0711.4572 .

[58] J. P. McKean, L. E. H. Godfrey, S. Vegetti, M. W. Wise, R. Morganti, M. J. Hardcastle, D. Rafferty, J. Anderson, I. M. Avruch, R. Beck, et al., MNRAS 463, 3143 (2016).

[59] A. Pandya, Z. Zhang, M. Chandra, and C. F. Gammie, ApJ 822, 34 (2016), 1602.08749.

[60] C. L. Carilli, J. D. Kurk, P. P. van der Werf, R. A. Perley, and G. K. Miley, AJ 118, 2581 (1999).

[61] A. S. Wilson, A. J. Young, and P. L. Shopbell, The Astrophysical Journal Letters 544, L27 (2000), URL http://stacks.iop.org/1538-4357/544/i=1/a=L27

[62] L. Huang and R. V. Shcherbakov, MNRAS 416, 2574 (2011), 1106.1630.

[63] C. L. Carilli, R. A. Perley, and J. H. Dreher, ApJ 334, L73 (1988).

[64] J. W. Dreher, C. L. Carilli, and R. A. Perley, ApJ 316, 611 (1987).

[65] D. P. Rayner, R. P. Norris, and R. J. Sault, Monthly Notices of the Royal Astronomical Society 319, 484 (2000), URL http://dx.doi.org/10.1111/j.1365-8711.2000.03854.x. 